\title{
Global Safety Database Summary of COVID-19-Related Drug Utilization-Safety Surveillance: A Sponsor's Perspective
}

\author{
Elena Beyzarov ${ }^{1}$ (D) $\cdot$ Yan Chen $^{1} \cdot$ Rob Julg $^{2} \cdot$ Karen Naim $^{1} \cdot$ Jigna Shah $^{1} \cdot$ William W. Gregory $^{1} \cdot$ Ayman Ayoub $^{1}$. \\ Patrick Caubel $^{1}$
}

Accepted: 15 December 2020 / Published online: 22 December 2020

(c) The Author(s) 2020

\begin{abstract}
Introduction Evidence-based clinical data on coronavirus disease 2019 (COVID-19) pharmacotherapies are scarce. Objective This study documented and characterized COVID-19 cases reported in individuals receiving treatment with Pfizer pharmaceutical products and cases that reported use of Pfizer pharmaceutical products for COVID-19 treatment.

Methods This retrospective observational review leveraged the Pfizer safety database containing adverse event data collected in association with use of Pfizer products between 1 October, 2019, and 25 June, 2020; the database includes worldwide adverse event data from various sources. Selected Medical Dictionary for Drug Regulatory Activities (MedDRA ${ }^{\circledR}$ ) Preferred Terms and subsequent clinical review were used to characterize COVID-19 cases.

Results Over 1500 relevant cases were identified over an 8-month period. In cases that reported COVID-19, immunosuppressant/immunomodulating agents, followed by anticoagulant/antithrombic agents and corticosteroids, were the most frequently reported agents. The frequent reporting of immunosuppressant/immunomodulating agents among cases of COVID-19 suggests increased vulnerability to infection among treated patients, either because of immunosuppressive effects of certain agents or the nature of the underlying treated condition. In cases involving off-label pharmacotherapy use for the treatment of COVID-19-related conditions, the most frequently reported therapeutic classes included antibiotics, antimalarial agents, antivirals/antiretroviral agents, immunosuppressant/immunomodulating agents, corticosteroids, anticoagulants, and immunoglobulin/interferons. The most frequently reported pharmacotherapeutic agents were azithromycin and chloroquine/hydroxychloroquine, followed by lopinavir-ritonavir, ceftriaxone, and tofacitinib. The most frequently reported clinical adverse events associated with azithromycin (as sole therapy or combined with chloroquine/hydroxychloroquine) include electrocardiogram QT prolonged, drug interaction, hepatitis, diarrhea, and hepatitis acute. Regarding cardiac-related events, 19\% (120/645) of azithromycin cases reported events associated with QT prolongation/torsade de pointes (which included seven fatal cardiac events). The most frequently reported clinical adverse events associated with other commonly used agents are also presented. Conclusions This pharmacovigilance surveillance study provides a unique characterization of cases in which a broad range of pharmaceutical products was reported in relation to COVID-19.
\end{abstract}

\section{Introduction}

Information regarding new treatment options and identification of existing treatments that may be repurposed for severe acute respiratory syndrome coronavirus $2 /$ coronavirus disease 2019 (SARS-CoV-2/COVID-19) therapy remains

Elena Beyzarov

Elena.Beyzarov@pfizer.com

$1 \quad$ Worldwide Medical and Safety, Worldwide Research and Development, Pfizer Inc, 100 US-206, Peapack, NJ 07977, USA

2 Information Management Operations Center of Excellence, Global Product Development Pfizer Inc, Peapack, NJ, USA scarce. Accordingly, clinicians must navigate treatment decisions in the absence of robust evidence-based clinical data. Given the broad range of therapeutic classes being utilized for COVID-19 treatment, systematic monitoring of these treatments and identification of important clinical events at the population level are paramount to patient safety.

The World Health Organization designated COVID-19 as a global pandemic on 11 March, 2020; a national emergency was then declared in the USA on 13 March, 2020. A weekly COVID-19 surveillance program was urgently initiated by Pfizer on 16 March, 2020, using data from the Pfizer safety database and routine pharmacovigilance. The objective of this retrospective observational study is to document and characterize COVID-19 cases reported in individuals 


\section{Key Points}

This retrospective study characterized COVID-19 cases reported in individuals receiving Pfizer pharmacotherapies and cases reporting Pfizer product use for COVID19 treatment.

In cases reporting COVID-19, immunosuppressant/ immunomodulating agents, anticoagulant/antithrombic agents, and corticosteroids were the most frequently reported pharmacotherapies; antibiotics and antimalarial agents were the two most commonly reported off-label pharmacotherapeutic classes.

Multiple pharmacotherapies are currently used for COVID-19 treatment; however, evidence-based clinical data are scarce; systematic surveillance of treatment outcomes is necessary to inform ongoing treatment strategies and to ensure patient safety.

receiving treatment with pharmaceutical products and of cases reporting use of pharmaceutical products for COVID19 treatment.

\section{Methodology}

\subsection{Pharmacovigilance Database}

The Pfizer global safety database contains cases of adverse events (AEs) reported in association with Pfizer product use; annually, approximately 530,000 cases are reported. Data are collected from multiple sources, including published literature, marketing programs, which include various nonclinical study programs (e.g., XELSOURCE, Accredo Xeljanz Program, Caremark Xeljanz C\&P Program) interventional or noninterventional studies (regardless of causality), business partners, patients, and health authorities.

\subsection{Case Search and Identification}

A retrospective search was conducted of AEs reported between 1 October, 2019, and 25 June 2020 (based on the initial safety receipt date of cases). COVID-19 cases were identified using the following Medical Dictionary for Regulatory Activities (MedDRA ${ }^{\circledR}$; Version 23.0; 14 May, 2020, rerelease) Preferred Terms (PTs): asymptomatic COVID-19, COVID-19, COVID-19 pneumonia, suspected COVID-19, SARS-CoV-2 carrier, exposure to SARS-CoV-2, occupational exposure to SARS-CoV-2, coronavirus test, SARSCoV-1 test, SARS-CoV-1 test positive, SARS-CoV-2 test, SARS-CoV-2 test false negative, SARS-CoV-2 test positive,
COVID-19 prophylaxis, COVID-19 treatment, coronavirus infection, coronavirus test positive and COVID-19 immunization. Before implementation of the updated MedDRA ${ }^{\circledR}$ rerelease, historical cases were retrieved primarily using a text string search. ${ }^{1}$ These historical cases were continuously reviewed for follow-up information relevant to COVID-19 infections. Additionally, case processing efforts to update the coding of cases that were reported to the safety database prior to the MedDRA ${ }^{\circledR}$ update (on 4 May, 2020) were undertaken; as such, these historical cases currently include relevant coronavirus terms, based on the updated MedDRA ${ }^{\circledR}$ version 23.0.

Identified cases underwent clinical review for relevancy by two colleagues who conducted independent reviews and subsequently reached consensus. Cases were excluded if they involved patients who either received testing for COVID-19 with pending/negative results, reported COVID-19-related symptoms with no information suggesting actual infection, reported other types of coronavirus or (in the context of the COVID-19 outbreak) inquired about temporarily discontinuing medications (e.g., immunosuppressant agents), delayed appointments/procedures, and reported exacerbations of anxiety/panic disorders. The relevant cases were then categorized into two groups. One group included cases of COVID-19 (as AEs or as part of medical history) reported during treatment with pharmaceutical products; these cases are grouped and discussed below in the section on cases of COVID-19 infections. The other group included cases reporting pharmaceutical product use for COVID-19 treatment (and associated complications/concurrent infections). These cases are grouped and discussed below in the section on cases of off-label use for COVID-19 treatment. Of note, this group includes the total number of cases that reported off-label use for COVID-19-specific indications as well as cases that report unknown/nonspecific indications where, upon review, the agent was used for COVID-19 treatment. As a distinction, the number of cases that reported off-label use for COVID-19-specific indications only is included as well. Pharmaceutical products included Pfizer products and non-Pfizer products that were reported as co-treatments (within Pfizer's pharmacovigilance database).

The seriousness of each reported AE was determined by Pfizer physicians based on the International Council for Harmonisation guideline [1]; AEs were assessed as serious if the event was considered medically important or had an adverse clinical course (e.g., death, life threatening, required hospitalization, prolonged hospitalization). Clinical AEs were considered events of clinical nature (e.g., affecting

\footnotetext{
${ }^{1}$ Case narratives were searched for the following string words: CORONAVIRUS or CORONA VIRUS or CORONA-VIRUS or COVID19 or COVID 19 or COVID-19 or SARS-COV-2 or SARSCOV2 or SARS COV 2 or 2019-NCOV or 2019 NCOV.
} 
Fig. 1 Relevant cases reported weekly into the Pfizer safety database

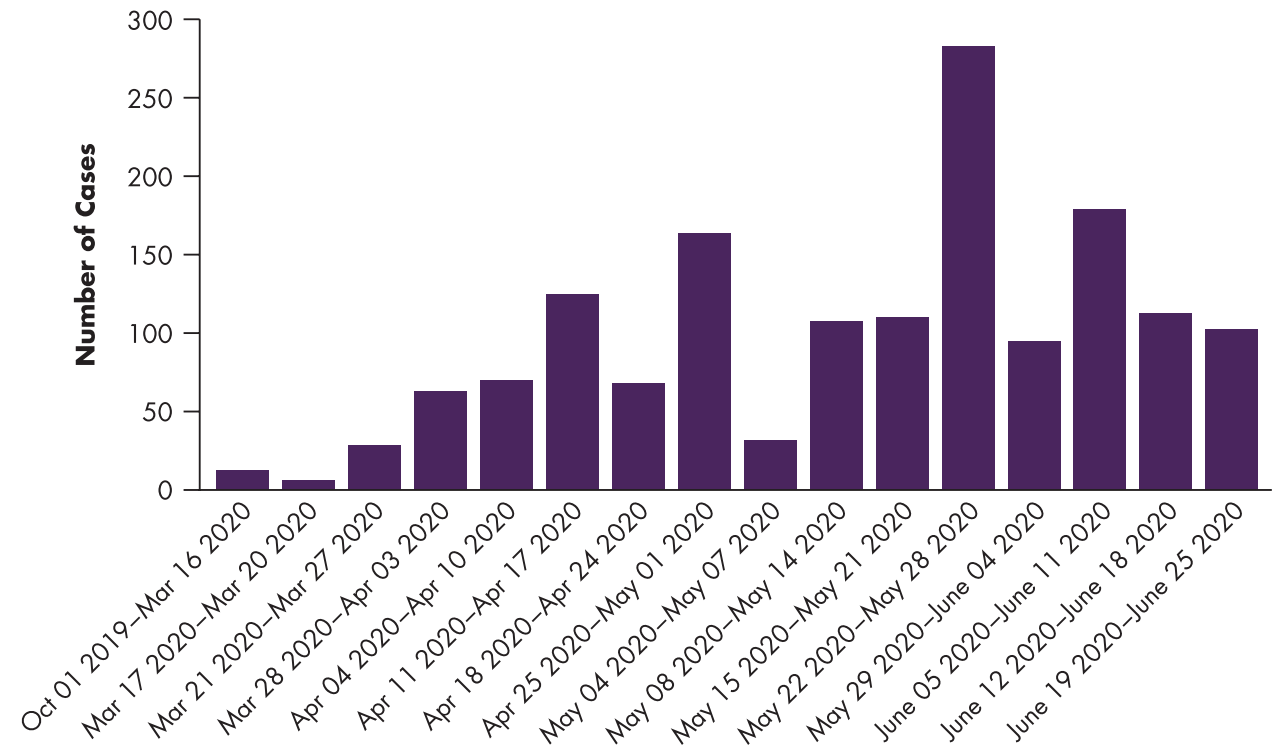

organ systems) rather than events encompassing certain MedDRA ${ }^{\circledR}$ derived conditions (e.g., medication errors, misuse, occupational exposure). Healthcare provider-reported cases were considered to be medically confirmed.

\section{Results}

\subsection{Overall Summary of Cases}

As of 25 June, 2020, 1508 relevant cases were identified (based on the aforementioned inclusion criteria described in the methodology section). The weekly case report is shown in Fig. 1. Cases included those reporting COVID19 -specific PTs as part of indications $(n=842)$, AEs $(n=$ $540)$, and medical history $(n=281)$; PTs could be reported in one or more fields. Overall, 602 cases reported COVID19 during treatment with pharmaceutical products and 910 cases reported pharmaceutical product use for COVID-19 treatment. Four cases reported COVID-19 as both AEs and treatment indications.

Cases were most frequently reported from the USA, France, and Spain. Among cases where age was reported ( $N=1093$; age not reported in 415 cases), the majority were adults; pediatric cases and elderly cases (adults aged $\geq 65$ years $)$ represented $1.5 \%(n=22)$ and $29 \%(n=433)$ of all cases, respectively. Overall, 46\% (694/1508) of total cases and $62 \%$ (268/433) of elderly cases reported one or more co-morbidities ${ }^{2}$ that may be considered potentially associated with either susceptibility to infection (i.e., use

\footnotetext{
${ }^{2}$ Considered to be relevant predisposing medical history (e.g., hypertension, autoimmune conditions, respiratory conditions, diabetes mellitus, cardiac conditions) and/or use of immunosuppressant/immunomodulating agents.
}

of immunosuppressant/immunomodulating agents for autoimmune or oncology indications) and/or with potentially adverse outcome of infection (relevant medical history; Fig. 2) [2]. Currently, there are limited data regarding the impact of many underlying medical conditions and whether they increase the risk for severe illness from COVID-19. As such, this information is evolving and additional epidemiologic studies are needed to further elucidate predisposing risk factors associated with susceptibility to (and worsening outcomes from) COVID-19 infection.

Overall, 119 cases $(7.9 \%)$ reported 223 events resulting in fatal outcomes; $61 \%$ of these cases involved elderly patients, with greater reporting of male (73 cases) vs female (42 cases) patients (four cases did not provide gender information). The majority (91/119) of these cases included one or more co-morbidities that may be considered potentially associated with either susceptibility to infection (i.e., use of immunosuppressant/immunomodulating agents for autoimmune or oncology indications) and/or associated with potentially /adverse outcome of infection (relevant medical history) [2]. Of these fatal cases, 60\% (71/119) reported fatal outcomes in association with COVID-19-specific PTs. In the remaining cases, fatal events appeared to be consistent with underlying infections or concurrent complications (e.g., cardiorespiratory arrest/cardiac complications, cerebral hemorrhage, neoplasm progression). Several of these cases also reported fatal acute respiratory distress/failure, which may be related to COVID-19 (but not inclusive of COVID-19-specific PTs).

\subsection{Cases of COVID-19 Infections}

A total of 602 cases reported COVID-19 during treatment with pharmaceutical products; these included both suspected 


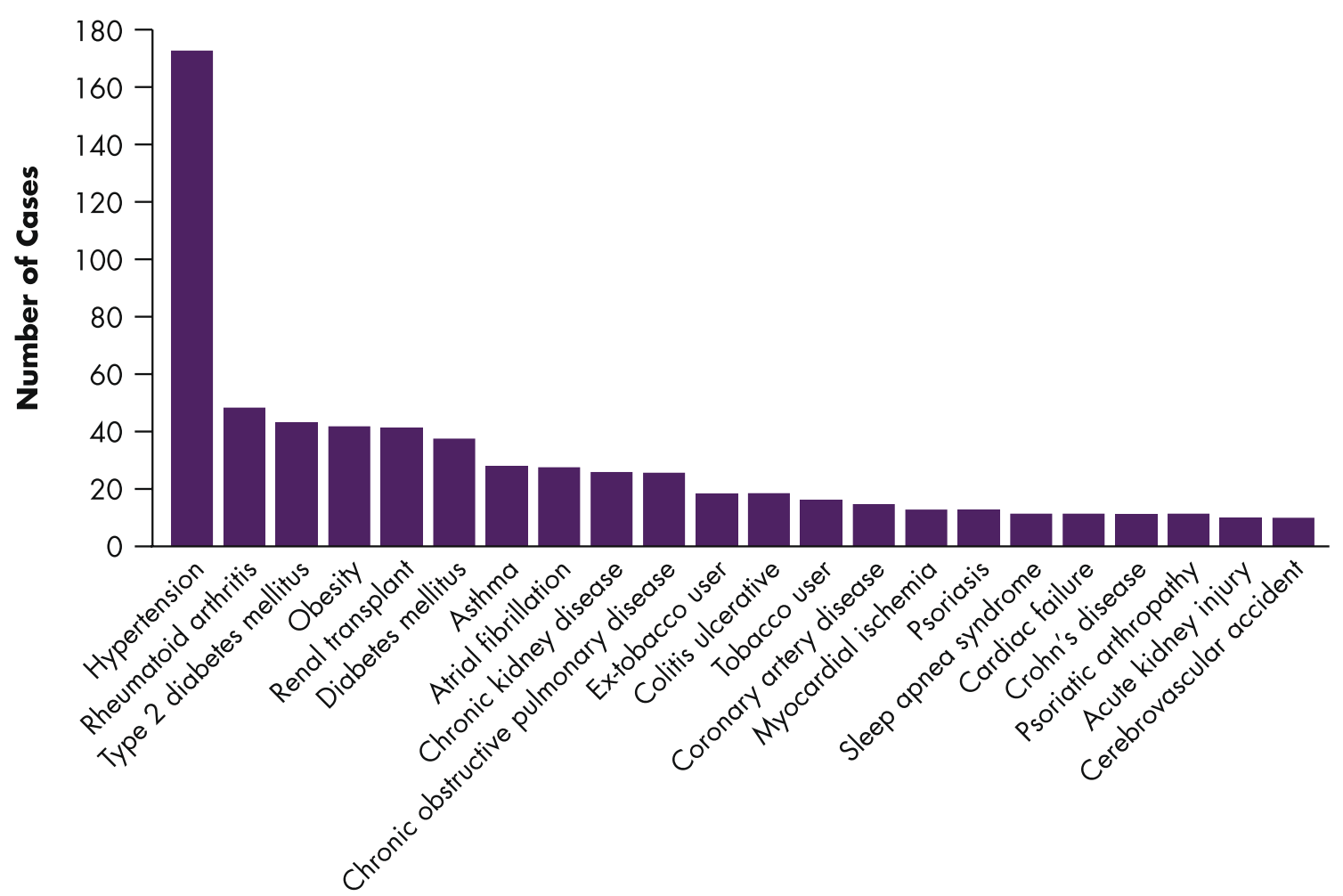

Fig. 2 Most commonly (ten or more cases) reported cumulative relevant medical history

and diagnostic laboratory test-confirmed COVID-19 cases. COVID-19-specific PTs were reported in 539 cases. Of these 539 cases, $85 \%$ (456/539) of cases were considered serious and 208 cases either required hospitalization or prolonged hospitalization. The $\geq 65$ year age group, followed by the 51- to 64-year age group, were the most frequently reported in association with these COVID-19-specific PTs. The cases were similarly distributed among both sexes.

Of the 539 cases reporting COVID-19-specific PTs, reporter causality was provided in 104 cases; most of which originated from clinical trials. In these 104 cases, events were possibly related to pharmaceutical products in 19 cases and unrelated in 89 cases (four of the cases reported both related and unrelated events). The most frequently reported pharmaceutical agents are summarized in Table 1 and discussed below.

\subsubsection{Immunosuppressant/Immunomodulating Agents}

Overall, 65\% (390/602) of cases reported use of immunosuppressant/immunomodulating agents primarily for autoimmune-related conditions or malignancies (these cases did not involve COVID-19 treatment indications). Among these, 59\% (353/602) of cases reported 368 events coded to COVID-19-specific PTs during treatment. In the majority of these cases, latency from the start of immunosuppressant/immunomodulating therapy (or timing of the indication under treatment) to the reported COVID-19-specific events was not provided. Therefore, causality assessment regarding the association between immunosuppression (either related to the suspect agents or treatment indications) and COVID-19 infection is limited. The most frequently (ten or more cases) reported treatment indications (when known) included rheumatoid arthritis $(n=100)$, psoriatic arthropathy $(n=25)$, colitis ulcerative $(n=21)$, immunosuppressant drug therapy $(n=18)$, Crohn's disease $(n=13)$, psoriasis $(n=11)$, and breast cancer metastatic $(n=10)$. The most commonly (ten or more cases) reported immunosuppressant/ immunomodulating agents are summarized in Table 1.

\subsubsection{Anticoagulant/Antithrombic Agents (Including Apixaban and Enoxaparin)}

Overall, 21\% (124/602) of cases reported apixaban ${ }^{3}$ and enoxaparin use for prophylaxis of cerebrovascular accidents and for thrombosis-related indications; 78 cases reported 78

\footnotetext{
${ }^{3}$ Nine additional reported cases include use of blinded therapy (apixaban; aspirin) for prophylaxis of cerebrovascular accidents; use of apixaban in these cases is dependent on randomization.
} 
Table 1 Frequently reported pharmaceutical agents coded to coronavirus disease 2019 (COVID-19)-specific Medical Dictionary for Drug Regulatory Affairs (MedDRA ${ }^{\circledR}$ ) Preferred Terms

\begin{tabular}{lc}
\hline Pharmaceutical agent class/drug & Cases, $n$ \\
\hline Immunosuppressant/immunomodulating agents & 353 \\
Tofacitinib & 123 \\
Etanercept & 95 \\
Methotrexate & 39 \\
Palbociclib & 32 \\
Tacrolimus & 22 \\
Infliximab & 18 \\
Mycophenolate mofetil & 13 \\
Anticoagulant/antithrombic agents & 78 \\
Apixaban & 97 \\
Enoxaparin & 27 \\
Corticosteroids & 33 \\
Methylprednisolone & 29 \\
Prednisone/prednisolone & 25 \\
Dexamethasone & 8 \\
Ciclesonide & 1 \\
Mometasone & 1 \\
\hline
\end{tabular}

events coded to COVID-19-specific PTs during treatment. The majority $(65 / 124)$ involved elderly patients or those with relevant medical history (e.g., hypertension, cardiac conditions, malignancy, diabetes mellitus).

\subsubsection{Corticosteroids}

Overall, 9\% (57/602) of cases reported the use of corticosteroids as part of treatment regimens for various inflammatory/autoimmune-based conditions, organ transplant-related immunosuppression, or malignancy. Reported agents are included in Table 1. Among these cases, 33 cases reported 34 events coded to COVID-19-specific PTs during treatment.

\subsection{Cases of Off-Label Use for COVID-19 Treatment}

A total of 910 cases $^{4}$ involved off-label pharmacotherapy use for COVID-19; 842 of these cases reported off-label use for COVID-19-specific indications. In these cases, various antimicrobials (e.g., antibiotics, antiretrovirals, antivirals) and other supportive therapy (e.g., corticosteroids, immunoglobulin) were employed.

Overall, $8 \%$ (72/910) of cases co-reported other infections (e.g., pneumonia, bronchitis, bacterial superinfection,

\footnotetext{
4 Total case count includes cases that reported off-label use for COVID-19-specific indications as well as cases that report unknown/ nonspecific indications where, upon review, the agent was used for COVID-19 treatment.
}

urinary tract infection, aspergillus infection, sepsis) as part of treatment indications. Generally, antimicrobials were initiated empirically in patients presenting with lower respiratory tract infections, such as pneumonia (before receiving coronavirus test results), or were used off-label for the treatment of documented COVID-19 and underlying complications. The majority of agents used for COVID-19 treatment involved concomitant treatment (commonly consisting of more than two drug combinations) used for COVID-19 and concurrent complications. As such, the reported clinical AEs are confounded by (and potentially associated with) multiple agents. The most frequently reported therapeutic classes and agents (ten or more cases) used for COVID-19-specific indications are reported in Table 2 and discussed below.

\subsubsection{Azithromycin (as Sole Therapy or Combined with Chloroquine/Hydroxychloroquine)}

Azithromycin was the most commonly used antimicrobial agent and represented $71 \%$ (645/910) of cases (615 cases involving COVID-19-specific indications and 30 additional cases reporting unknown/nonspecific indications where the agent was used for COVID-19 treatment). The majority (84\% [541/645]) of these cases were spontaneous reports, most frequently originating from France (31\% [199/645 cases]).

The majority of cases involved use of azithromycin in combination with multiple other antimicrobial agents and supportive therapy; co-treatment drugs were involved in $69 \%(442 / 645)$ of cases. The most frequently ( $>20$ cases) reported co-suspect agents included hydroxychloroquine ( $n$ $=378$; chloroquine reported in six cases), ceftriaxone $(n=$ $99)$, lopinavir-ritonavir $(n=94)$, and tocilizumab $(n=41)$.

The vast majority ( $89 \%$ [573/645]) of cases involved offlabel use-related PTs (Off label use, Product use in unapproved indication, Drug ineffective for unapproved indication, Drug effective for unapproved indication, Product use issue), which were reported as single events in $43 \%$ (278/645) of cases. Clinical AEs were reported in 55\% (355/645) of cases; the most frequently (five or more cases) reported events are shown in Fig. 3. Regarding cardiacrelated events, $19 \%(120 / 645)$ of cases reported 126 events associated with QT prolongation/torsade de pointes (TdP).

Cardiac events relevant to QT prolongation/TdP within the 120 cases included the PTs electrocardiogram QT prolonged $(n=94)$, long QT syndrome $(n=19)$, ventricular tachycardia $(n=7)$, sudden death $(n=3), \operatorname{TdP}(n=2)$, and cardiac arrest $(n=1)$. When the clinical outcome was known, these serious events were reported as resolved/resolving ( $n=$ $89)$, fatal $(n=7)$, and not resolved and resolved with sequelae $(n=1$ each); permanent treatment withdrawal occurred in $65 \%(78 / 120)$ of cases. The vast majority (88\% [106/120]) of these cases reported concomitant/sequential treatment 
Table 2 Frequently reported off-label pharmaceutical agents used for coronavirus disease 2019 (COVID-19) treatment

\begin{tabular}{lc}
\hline Pharmaceutical agent class/drug & Cases, $n$ \\
\hline Antibiotics & 661 \\
Azithromycin & 615 \\
Ceftriaxone & 90 \\
Piperacillin-tazobactam & 16 \\
Meropenem & 12 \\
Antimalarial agents (hydroxychloroquine, chloroquine) & 404 \\
Antivirals/antiretroviral agents & 153 \\
Lopinavir-ritonavir ${ }^{a}$ & 126 \\
Ribavirin & 11 \\
Immunomodulating agents & 138 \\
Tofacitinib & 73 \\
Tocilizumab & 52 \\
Sarilumab & 10 \\
Corticosteroids & 39 \\
Methylprednisolone & 24 \\
Immunoglobulin/interferons & 15 \\
Anticoagulants & 12 \\
Antifungal agents & 5 \\
\hline
\end{tabular}

${ }^{a}$ Lopinavir and ritonavir prescribed either as a fixed-dose combination or as individual agents with azithromycin and hydroxychloroquine (and chloroquine in five cases), with several cases reporting an associated drug interaction PT. Upon further review, 65\% (78/120) of cases involved patients of advanced age ( $\geq 65$ years; posing increased susceptibility to drug-associated effects on QT interval) or relevant medical history (e.g., hypertension, cardiac conditions, diabetes, renal disease, hypothyroidism). The two cases reporting TdP involved a medical history of possible congenitally prolonged QT interval in one case and advanced patient age (79 years) in the other.

Hydroxychloroquine and chloroquine were the second most commonly used agents for COVID-19 treatment, representing 44\% (404/910) of cases and (as with azithromycin) most frequently originating from France (39\% [159/404 cases]). Because most of these cases involved co-treatment with azithromycin (as noted above), the reported AEs are confounded by both agents and generally follow a pattern similar to that observed in association with azithromycin.

\subsubsection{Lopinavir-Ritonavir (Either as a Fixed-Dose Combination or as Individual Agents)}

Lopinavir-ritonavir treatment was reported in 14\% (129/910) of cases (126 cases that involved COVID-19-specific indications and three additional cases of unknown/nonspecific

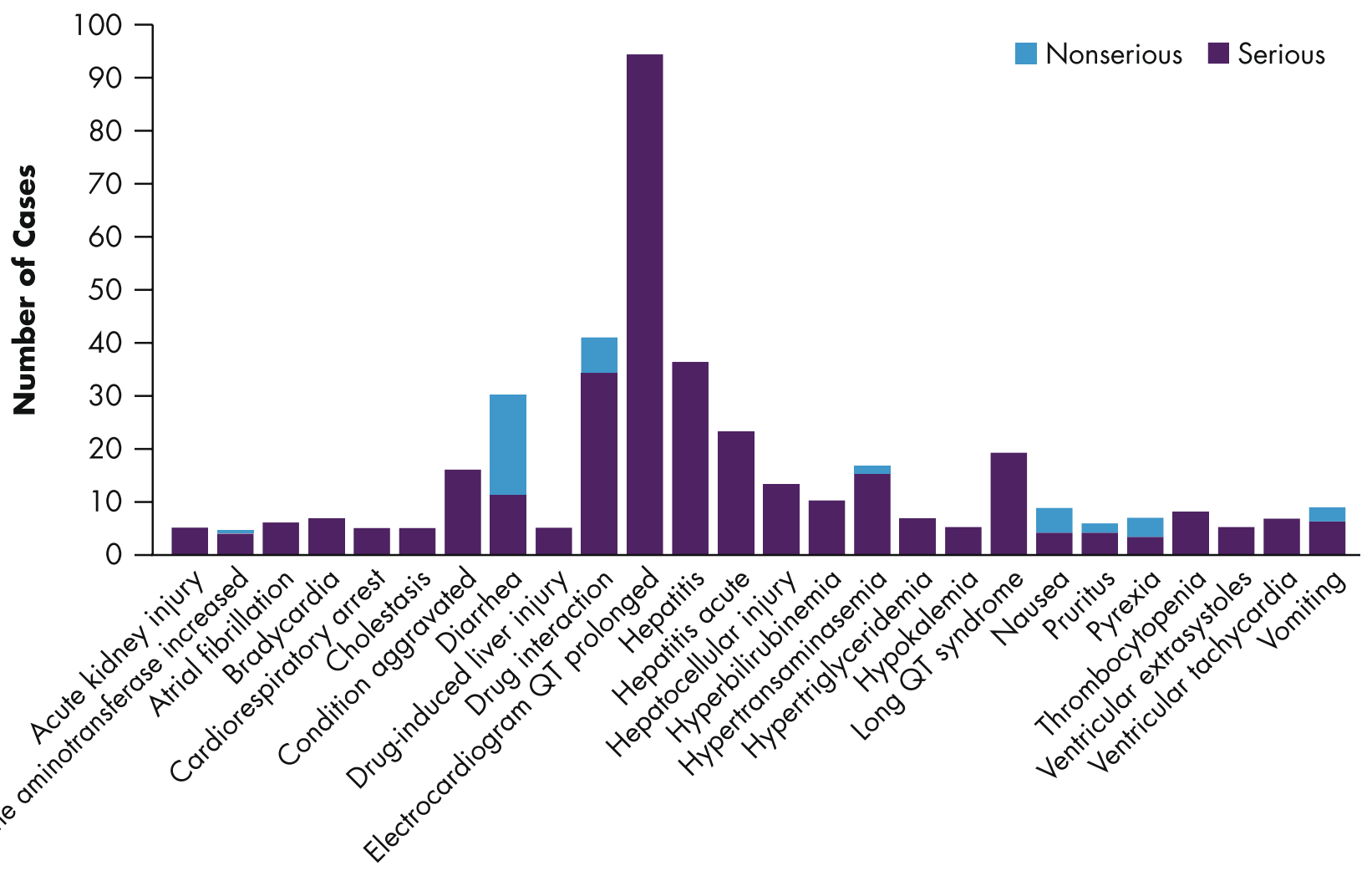

Fig. 3 Most frequently (five or more cases) reported cumulative clinical preferred terms and event seriousness associated with use of azithromycin for treatment of coronavirus disease 2019 (COVID-19) infections 


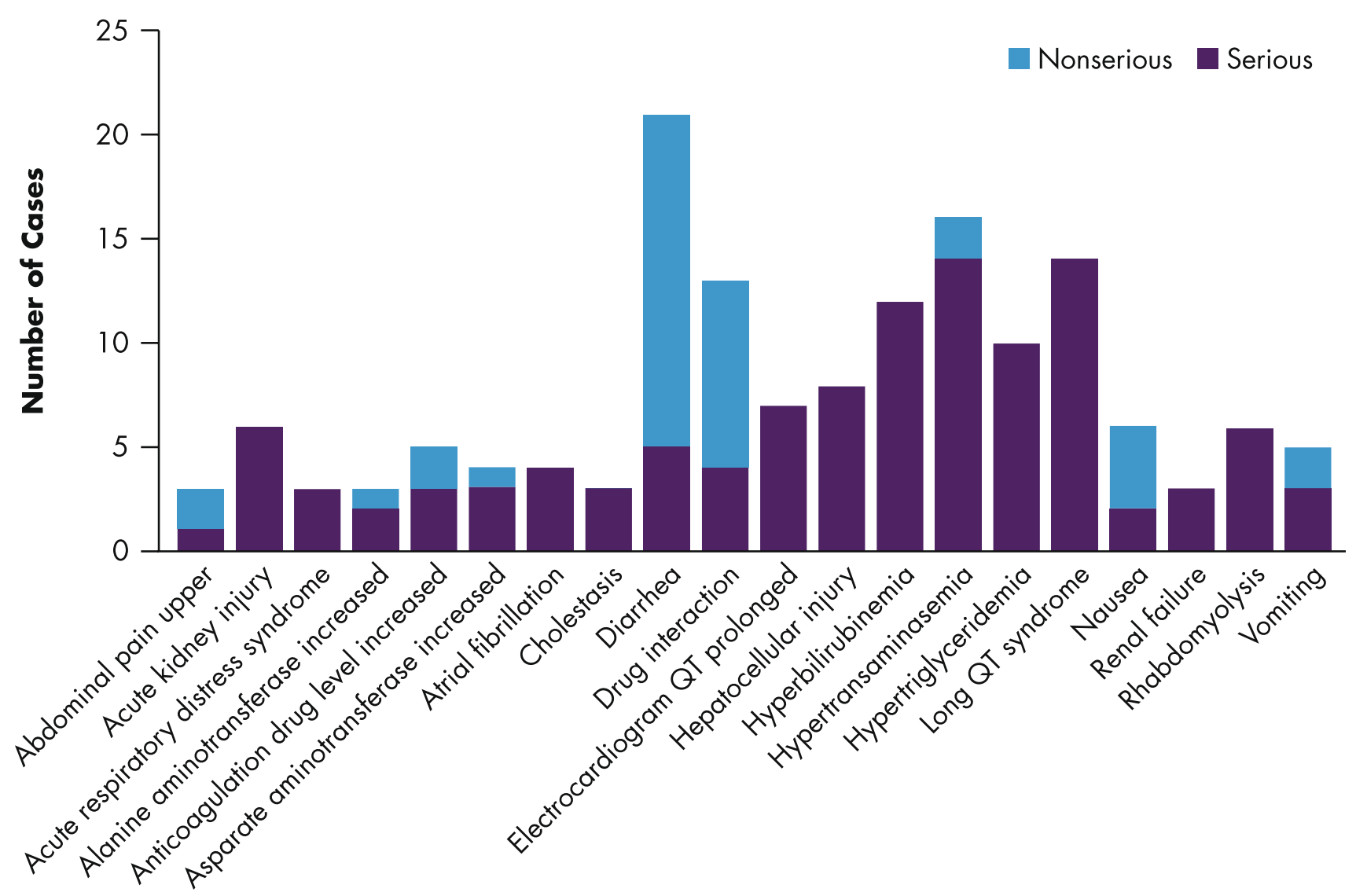

Fig. 4 Most frequently (three or more cases) reported cumulative clinical preferred terms and event seriousness associated with use of lopinavirritonavir for treatment of coronavirus disease 2019 (COVID-19) infections

indications where the agent was used for COVID-19 treatment; Table 2). Lopinavir-ritonavir was typically prescribed in combination with multiple other antimicrobial agents and supportive therapy; co-treatment drugs were involved in all these cases. The majority (92\% [119/129]) of cases reported numerous ( $n=237$ ) clinical AEs (Fig. 4); the most frequent $(n>10)$ included diarrhea $(n=21)$, hypertransaminasemia $(n=16)$, long QT syndrome $(n=14)$, drug interaction $(n=$ $13)$, hyperbilirubinemia $(n=12)$, and hypertriglyceridemia $(n=10)$. The majority of these events were considered serious, leading to permanent treatment discontinuation.

\subsubsection{Ceftriaxone}

Ceftriaxone was reported in 12\% (112/910) of cases (90 cases involving COVID-19-specific indications and 22 additional cases of unknown/nonspecific indications where the agent was used for COVID-19 treatment). Ceftriaxone was typically prescribed in combination with multiple other antimicrobial agents and supportive therapy; co-treatment drugs were involved in all of these cases. The majority $(75 \%$ [84/112]) of cases reported numerous mostly serious clinical AEs ( $n=151)$; the most frequent (five or more cases) included hepatitis ( $n=17$ ); condition aggravated and hypertransaminasemia ( $n=7$ each); diarrhea, hepatitis acute, and hypertriglyceridemia ( $n=6$ each); and hyperbilirubinemia and long QT syndrome ( $n=5$ each).

\subsubsection{Tofacitinib}

Tofacitinib was reported in $8 \%(73 / 910)$ of cases for COVID-19-specific indications (the majority of which were poorly documented and potentially solicited); 99\% (72/73) of cases reported off-label use-related PTs as the sole events. One case co-reported a clinical AE (pancreatitis acute) in a patient who was concurrently treated with several other immunosuppressive agents for dermatomyositis.

\section{Discussion}

This retrospective safety surveillance study provides a comprehensive review of the use of Pfizer's pharmaceutical products in relation to COVID-19 as reported to the Pfizer global pharmacovigilance database. Overall, 1508 relevant cases were identified between 1 October, 2019, and 25 June, 2020. 
The greatest number of cases were reported in the USA, reflecting the high burden of COVID-19 in the country [3]. France reported the second highest case count, which was mostly driven by azithromycin off-label use. The number of cases reported weekly and the characteristics of the cases are consistent with generally observed population-level trends. The majority of cases (70\%) occurred in adult patients, and $<2 \%$ of cases involved pediatric patients. Notably, nearly $50 \%$ of the total cases and $>60 \%$ of elderly cases involved one or more predisposing risk factors for infection/adverse outcome of infection, consistent with the profile of individuals at high risk for poor COVID-19 outcomes [2].

This study revealed that immunosuppressant agents were the most frequently reported pharmaceutical products (reported in 390 cases) among cases of COVID-19 infection/ pneumonia, suggesting increased vulnerability to infection among treated patients, either because of immunosuppressive effects of certain agents or the nature of the underlying treated condition. Of note, these 390 cases included indications involving autoimmune-related conditions or malignancies and did not involve any COVID-19 treatment indications (e.g., patients did not receive these agents as preventative treatment or post-infection with SARS$\mathrm{CoV}-2)$. Although viral infection is a known risk associated with immunosuppressant use, the observations of this study raise the questions of whether the risk of acquiring COVID-19 is increased among patients receiving immunosuppressants and whether treatment impacts the clinical course and outcome. However, as most of the reported cases lack associated exposure data, it is beyond the scope of this study to draw appropriate conclusions; observational studies leveraging data collected from existing healthcare databases and newly established COVID-19 registries are needed to address these questions. In further recognizing the need for maintaining a global safety database of drugs used for the management of COVID-19, a consortium formed by regulatory authorities and national health agencies, as well as a framework for systemic risk-benefit assessment tools, would be beneficial in centralizing the accumulating pharmacovigilance-related information pertaining to COVID-19.

Cases originating from post-marketing data and published literature have reported use of immunosuppressants/ immunomodulators, such as Janus kinase inhibitors (tofacitinib), for off-label treatment of COVID-19 pneumonia [4]; however, information relating to safety and therapeutic outcomes is limited, preventing further assessment at this time. Published case studies detailing management of COVID-19 potentially support the concept of baseline immunosuppressive therapy to prevent (or not worsen) COVID-19 severity by attenuating the increased cytokine response. For example, in a case study of a dual organ transplant recipient who experienced a mild COVID-19 course, despite the presence of numerous risk factors and an immunosuppressed status, the immunosuppressant mycophenolate mofetil was held while tacrolimus and prednisone were continued during the course of infection [5]. Another case study described a patient with ulcerative colitis who contracted COVID-19 during tofacitinib treatment; in this case, treatment was continued uninterrupted [6]. The patient's respiratory symptoms resolved several days after symptom onset with no residual symptoms after 2 weeks. The authors noted that while the role of tofacitinib in the patient's recovery was not conclusive, it did not appear to be a confounding factor with regard to COVID-19 severity. Another case study found that various disease-modifying antirheumatic drugs may affect COVID19 course and prognosis differently [7]. Sharmeen and colleagues suggested that while pretreatment with rituximab or secukinumab may worsen the viral disease and its related complications, etanercept, hydrochloroquine, or tocilizumab may be associated with a milder disease course (supporting a positive role of selective immune inhibition, such as anti-interleukin-6 with tocilizumab). Finally, in other case studies of patients with COVID-19 receiving immunosuppressive therapy, no significant complications were found during the infection course. Baseline immunosuppression was continued in a study of organ transplant recipients [8], and in another case study, the incidence of COVID-19 hospitalization among patients with immune-mediated inflammatory disease was consistent with that observed in the general population [9]. While this balanced approach of potentially continuing baseline immunosuppressive therapy (albeit with dose reductions in some instances) has been associated with positive outcomes in some patients, conclusions should be cautiously interpreted because of the small sample sizes of the cited case studies and our dataset; definitive conclusions await randomized clinical studies.

Similar to immunosuppressant/immunomodulating agents, the association of corticosteroids with COVID-19 events is likely driven by the immunosuppressive effects of treatment. Based on treatment indications among COVID19 cases, corticosteroids were possibly used as maintenance therapy, exposing patients to the potential immunosuppressive effects associated with long-term corticosteroid use. Conversely, corticosteroids have also been used for the treatment of COVID-19 (albeit typically acutely/short term). Recently, preliminary results from the RECOVERY trial have demonstrated that the use of dexamethasone can potentially lower 28-day mortality among patients with more severe COVID-19 conditions (e.g., those who were receiving either oxygen support or invasive mechanical ventilation at baseline) [10]. Anticoagulant/antithrombic agents (apixaban, enoxaparin) are also frequently reported in association with COVID-19 events. In patients treated with these agents, vulnerability to infection with SARS-CoV-2 and/or progression to COVID-19 is likely attributed to advanced age and relevant medical history rather than anticoagulant/ 
antithrombic use. There are several literature cases retrieved from the Pfizer safety database discussing the interaction between antiviral therapies and direct oral anticoagulants (DOACs) on account of both being substrates of the P-glycoprotein or cytochrome P450-based metabolic pathways [11]. The authors cautioned that concomitant DOAC and antiviral drug administration is associated with a potentially sharp increase in DOAC anticoagulant plasma concentrations, possibly increasing hemorrhagic risk. While our cases did not report bleeding-related AEs, other associated events (increased anticoagulation drug concentration and interaction) were co-reported and described as an alarming increase in DOAC plasma concentrations compared with those seen before hospitalization.

In addition to the aforementioned immunosuppressants and anticoagulants, other therapeutic classes have been reported for the treatment of COVID-19. Azithromycin is one of the most frequently reported treatments. Currently, numerous clinical studies have been conducted to evaluate the potential benefit/risk regarding use of azithromycin for the treatment of COVID-19 conditions; however, results are less conclusive [12-14]. In our analyses, the most frequently reported $\mathrm{AE}$ in cases involving the use of azithromycin for the treatment of COVID-19 was electrocardiogram QT prolongation; which in the majority of cases was assessed as either resolved or resolving at the time of reporting.

A review of the azithromycin cases that reported QT prolongation/TdP events suggests that these events likely occurred as a result of concomitant administration of azithromycin and hydroxychloroquine (and chloroquine to a much lesser extent), which were reported in approximately $90 \%$ of these cases. Another consideration for the observed increase in QT prolongation cases is the treated condition (COVID-19), which is known to significantly impact vulnerable populations with possibly pre-existing cardiac risk factors, as well as exhibiting potential independent viral effects on the myocardium $[15,16]$. Subsequent disproportionality analyses (data on file) revealed substantial observed-to-expected reporting frequencies involving the azithromycin/hydroxychloroquine combination and QT prolongation-related events; these exceeded those associated with individual reporting of azithromycin or hydroxychloroquine. This finding is consistent with a recently published retrospective analysis of 3737 patients with COVID-19 treated with hydroxychloroquine/azithromycin and other regimens in France [17]. In that study, QTc prolongation was observed in 25 patients, most of whom were treated with azithromycin-hydroxychloroquine $(n=20)$. Similar to the small number of TdP cases reported in our study ( $n$ $=2$ ), no TdP or sudden death cases were observed in the French study. More studies with formal comparisons may be needed to further characterize the cardiovascular safety profile of the azithromycin/hydroxychloroquine combination vs individual treatments in the setting of COVID-19, to inform healthcare providers in clinical practice. Nevertheless, Pfizer issued a communication informing relevant regulatory agencies regarding the safe use of azithromycin for COVID-19 treatment in countries where azithromycin is marketed. This resultant risk management measure underscores the role and value of spontaneous case reporting as a cornerstone of pharmacovigilance.

Our analyses include several strengths. First, the large volume of data analyzed ( $>1500$ cases) allowed for meaningful characterization and interpretation of reporting trends. Second, bias was minimized because most cases were reported spontaneously $(<10 \%$ of cases originated from solicited sources such as various company-sponsored marketing programs). Third, our analyses encompassed various clinical settings (e.g., outpatient, hospitalization [required or prolonged in 25\% [380/1508] of cases, when information was available], clinical trials) and patient characteristics (e.g., age, sex, health status), thus enhancing the generalizability of our results. Additionally, pharmaceutical agent use reported into the Pfizer safety database includes both Pfizer and non-Pfizer products, thereby providing a holistic portrait of therapies used in reported COVID-19 cases. Finally, as a large percentage $(80 \%)$ of retrieved cases were medically confirmed, this optimized the clinical credibility and quality of reported case information.

Several limitations that are inherent to post-marketing AE reporting should be considered when interpreting these data. First, underreporting occurs because pharmacovigilance reports are submitted voluntarily. Many factors influence AE reporting, which could create reporting bias. As this yields reporting proportions instead of incidence rates, it is not possible to make between-drug comparisons using these proportions. Second, the reported cases are mainly related to AEs; thus, information regarding efficacy and outcome is lacking or limited. Finally, in some reports, important clinical information (e.g., severity status of COVID-19 events) and causality assessment (owing to the majority of the cases originating from spontaneous sources) may be missing or incomplete, with no follow-up or outcome information available.

\section{Conclusions}

There were 1508 relevant cases identified over an 8-month period. In cases that reported COVID-19, immunosuppressant/immunomodulating agents, followed by anticoagulant/ antithrombic agents and corticosteroids, were the most frequently reported pharmaceutical products used. In cases involving off-label pharmacotherapy use for the treatment of COVID-19-related conditions, the most frequently reported therapeutic classes included antibiotics, antimalarial agents, 
antivirals/antiretroviral agents, immunosuppressant/immunomodulating agents, corticosteroids, anticoagulants, and immunoglobulin/interferons. The most frequently reported pharmacotherapeutic agents were azithromycin and chloroquine/hydroxychloroquine. Review of the AEs in association with off-label treatment of COVID-19 has not identified any new safety concerns beyond the known safety profile of these agents. Continuous pharmacovigilance including monitoring, tracking, and comparative analyses of the safety profile of these agents when used in the COVID-19 patient population (vs general population within labeling) may be warranted.

Evidence-based clinical data related to COVID-19 treatment and information gathered via pharmacovigilance activities (particularly via spontaneous reporting) are vital to patient treatment and safety. This retrospective pharmacovigilance surveillance study provides meaningful characterization of pharmaceutical product use in relation to COVID-19; such information could be critical in informing the ongoing care of patients with COVID-19.

Acknowledgements Manuscript review and additional associated analyses provided by Joanne L. Leaney, PhD, Marysol Diego Bishara, PharmD, Kevin T. Cornetto, PharmD, Yu Tracy Gao, MD, MBA, Jaymini Patel, PharmD, Erast Pohorylo, PharmD, Anna Portnoy, PharmD, Jackit Tungmun, R.Ph., and Ashwin Ravi, MBBS, MD (Pharmacology) of Pfizer Inc. Editorial/medical writing support was provided by Srividya Ramachandran, $\mathrm{PhD}$, and Tricia Newell, $\mathrm{PhD}$, of ICON plc (North Wales, PA, USA), and was funded by Pfizer Inc.

\section{Declarations}

Funding This work was supported by Pfizer Inc.

Conflict of interest Elena Beyzarov, Yan Chen, Rob Julg, Karen Naim, Jigna Shah, William W. Gregory, Ayman Ayoub, and Patrick Caubel are employees of Pfizer Inc. and may hold stock or stock options.

Ethics approval The analyses for this study were conducted retrospectively using deidentified patient data; as such, this study was deemed exempt by the institutional review board and informed consent was not required.

\section{Consent to participate Not applicable.}

Consent for Publication Not applicable.

Availability of data and material Upon request, and subject to certain criteria, conditions, and exceptions (see https://www.pfizer.com/scien ce/clinical-trials/trial-data-and-results for more information), Pfizer will provide access to individual de-identified participant data from Pfizer-sponsored global interventional clinical studies conducted for medicines, vaccines, and medical devices (1) for indications that have been approved in the USA and/or European Union or (2) in programs that have been terminated (i.e., development for all indications has been discontinued). Pfizer will also consider requests for the protocol, data dictionary, and statistical analysis plan. Data may be requested from Pfizer trials 24 months after study completion. The de-identified participant data will be made available to researchers whose proposals meet the research criteria and other conditions, and for which an exception does not apply, via a secure portal. To gain access, data requestors must enter into a data access agreement with Pfizer.

Code availability No specific custom code was created for the study. We utilized an SAP Business Objects (Desktop Intelligence Version 12.6.2.1782) based application to analyze the study data. For more details, please contact the corresponding author.

Author contributions All authors met the below criteria for authorship: substantial contributions to the conception or design of the work; or the acquisition, analysis, or interpretation of data for the work; and drafting the work or revising it critically for important intellectual content; and final approval of the version to be published; and agreement to be accountable for all aspects of the work in ensuring that questions related to the accuracy or integrity of any part of the work are appropriately investigated and resolved.

Open Access This article is licensed under a Creative Commons Attribution-NonCommercial 4.0 International License, which permits any non-commercial use, sharing, adaptation, distribution and reproduction in any medium or format, as long as you give appropriate credit to the original author(s) and the source, provide a link to the Creative Commons licence, and indicate if changes were made. The images or other third party material in this article are included in the article's Creative Commons licence, unless indicated otherwise in a credit line to the material. If material is not included in the article's Creative Commons licence and your intended use is not permitted by statutory regulation or exceeds the permitted use, you will need to obtain permission directly from the copyright holder. To view a copy of this licence, visit http://creativecommons.org/licenses/by-nc/4.0/.

\section{References}

1. European Medicines Agency. Guideline on good pharmacovigilance practices (GVP): Annex I - Definitions (Rev 4); 9 Oct 2017. https://www.ema.europa.eu/en/documents/scientific-guideline/ guideline-good-pharmacovigilance-practices-annex-i-definition s-rev-4_en.pdf

2. Centers for Disease Control and Prevention. CDC coronavirus disease 2019 (COVID-19): people with certain medical conditions. https://www.cdc.gov/coronavirus/2019-ncov/need-extra -precautions/people-with-medical-conditions.html. Accessed 14 Sep 2020.

3. Centers for Disease Control and Prevention. CDC COVID data tracker. https://covid.cdc.gov/covid-data-tracker/. Accessed 26 Oct 2020.

4. Walz L, Cohen AJ, Rebaza AP, Vanchieri J, Slade MD, Cruz CSD, et al. JAK-inhibitor and type I interferon ability to produce favorable clinical outcomes in COVID-19 patients: a systematic review and meta-analysis. Res Sq. 2020:rs.3.rs-64782.

5. Hsu JJ, Gaynor P, Kamath M, Fan A, Al-Saffar F, Cruz D, et al. COVID-19 in a high-risk dual heart and kidney transplant recipient. Am J Transplant. 2020;20:1911-5.

6. Jacobs J, Clark-Snustad K, Lee S. Case report of a SARS-CoV-2 infection in a patient with ulcerative colitis on tofacitinib. Inflamm Bowel Dis. 2020;26:e64.

7. Sharmeen S, Elghawy A, Zarlasht F, Yao Q. COVID-19 in rheumatic disease patients on immunosuppressive agents. Semin Arthritis Rheum. 2020;50:680-6.

8. Ning L, Liu L, Li W, Liu H, Wang J, Yao Z, et al. Novel coronavirus (SARS-CoV-2) infection in a renal transplant recipient: case report. Am J Transplant. 2020;20:1864-8. 
9. Haberman R, Axelrad J, Chen A, Castillo R, Yan D, Izmirly P, et al. Covid-19 in immune-mediated inflammatory diseases: case series from New York. N Engl J Med. 2020;383:85-8.

10. Recovery Collaborative Group, Horby P, Lim WS, Emberson JR, Mafham M, Bell JL, et al. Dexamethasone in hospitalized patients with Covid-19: preliminary report. N Engl J Med. 2020. https:// doi.org/10.1056/NEJMoa2021436.

11. Testa S, Prandoni P, Paoletti O, Morandini R, Tala M, Dellanoce $\mathrm{C}$, et al. Direct oral anticoagulant plasma levels' striking increase in severe COVID-19 respiratory syndrome patients treated with antiviral agents: the Cremona experience. J Thromb Haemost. 2020;18:1320-3.

12. Fiolet T, Guihur A, Rebeaud ME, Mulot M, Peiffer-Smadja N, Mahamat-Saleh Y. Effect of hydroxychloroquine with or without azithromycin on the mortality of coronavirus disease 2019 (COVID-19) patients: a systematic review and meta-analysis. Clin Microbiol Infect. 2020. https://doi.org/10.1016/j.cmi.2020.08.022.

13. Furtado RHM, Berwanger O, Fonseca HA, Correa TD, Ferraz LR, Lapa MG, et al. Azithromycin in addition to standard of care versus standard of care alone in the treatment of patients admitted to the hospital with severe COVID-19 in Brazil (COALITION II): a randomised clinical trial. Lancet. 2020;396:959-67.

14. Sekhavati E, Jafari F, SeyedAlinaghi S, Jamalimoghadamsiahkali S, Sadr S, Tabarestani M, et al. Safety and effectiveness of azithromycin in patients with COVID-19: an open-label randomised trial. Int J Antimicrob Agents. 2020;56:106143.

15. Zhu H, Rhee JW, Cheng P, Waliany S, Chang A, Witteles RM, et al. Cardiovascular complications in patients with COVID-19: consequences of viral toxicities and host immune response. Curr Cardiol Rep. 2020;22:32.

16. Madjid M, Safavi-Naeini P, Solomon SD, Vardeny O. Potential effects of coronaviruses on the cardiovascular system: a review. JAMA Cardiol. 2020;5:831-40.

17. Lagier JC, Million M, Gautret P, Colson P, Cortaredona S, GiraudGatineau A, et al. Outcomes of 3,737 COVID-19 patients treated with hydroxychloroquine/azithromycin and other regimens in Marseille, France: a retrospective analysis. Travel Med Infect Dis. 2020;36:101791. 